\title{
THE POTENTIAL ENERGY OF A CHARGE NEAR THE SURFACE OF THE SPHERICAL SEMICONDUCTOR MICROCRYSTAL AT THE PRESENCE OF INTERMEDIATE LAYER OF VARYING DIELECTRIC CONSTANT
}

\author{
V. I. Boichuk, R. Yu. Kubay \\ Drohobych Ivan Franko State Pedagogical University, Department of Theoretical Physics, \\ 24 Franko Str., Drohobych, UA-82100, Lviv Region, Ukraine \\ (Received April 27, 1999)
}

\begin{abstract}
In the present paper the effect of the semiconductor heterosystem interface (spherical microcrystal-matrix) on a charge patricle is studied. In a flat interfacial zone the case of existence of the intermediate layer with the dielectric constant as a function of distance is discussed. Using the Green's function the potential energy of a charge as a function of distance to the centre of microcrystal with various dielectric constants of the heterosystem is determined.
\end{abstract}

Key words: potential energy, spherical microcrystal, Green's function.

PACS number(s): 73.20.-r

\section{INTRODUCTION}

The problem of studying the influence of the crystal boundaries on the states of quasiparticles arises from the description of the processes taking place in two-media interface in fine semiconductor shells and microcrystals placed in the dielectric matrices.

Different aspects of this influence on the electron and exciton states have been examined in the papers [1-12]. In the case of the semiconductor surface to bound on vacuum or other medium with the dielectric constant less than that of the crystal, a resultant action gives rise to a flat interfacial zone ("dead" layer) that proves to scatter excitons. The "dead" layer model permits scattering spectra to be discussed in detail whose studying may provide useful information on interfacial states of a surface.

Using the classical procedure of image forces [1] the scattering potential to act on an exciton has been determined, and in adiabatic approximation by variational method a Schrödinger equation has been solved. As a result the bound energy of the electron-hole pair to depend on the distance of the centre of exciton mass to the crystal boundaries has been obtained. The region with positive value of the internal energy of an exciton is a "dead" layer itself.

In the case of a semiconductor of dielectric constant less than that of the bounding medium essentially new phenomena appear, namely the localization of excitons by image forces $[2,3]$. The phenomenon needs to be studied since exciton levels lying below the conduction band prove to considerably influence the edges of absorption spectrum.

Image forces play an important role in the formation of electron and exciton spectra in fine shells. Taking them in account helps to explain the dependence of excitation energy and bound energy upon shell thickness [4-6], the conditions of bound electron-surface and exciton-surface states $[7,8]$, the case of unstable electronic spectrum of the shell to bound on dielectic media of similar dielectric constants [9].

Analogous effects can be observed on studying electron and exciton states in the semiconductor microcrystal placed in dielectric matrices. As is shown in the papers $[10,11]$, in the case of glass matrix the bound electronsurface states can appear in the vicinity of the spherical semiconductor microcrystal surface. The potential of image forces strongly $[12,13]$ influences the excitation energy and bound energy of excitons in spherical microcrystals as well.

In most theoretical works taking account of the interaction of the interfacial zones with charge particles, dielectric permittivity is believed to assume various values to change stepwise transferring from one medium to another. However, it is understood that for each heterosystem there should exist a transitional layer of dielectric constant as a radial-dependent variable [14].

In the present paper the potential energy of a point charge particle has been obtained, when at the interface of the media the dependence $\varepsilon=\varepsilon(r)$ has been accounted for a spherical microcrystal placed in the matrix.

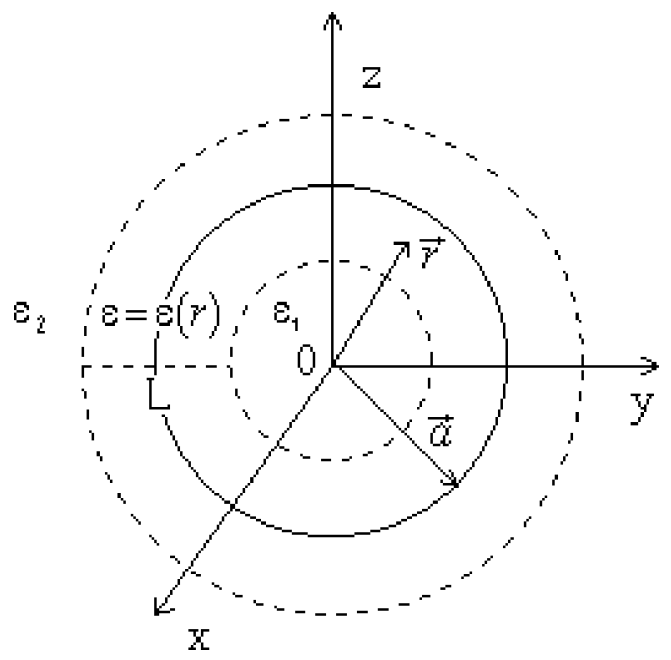

Fig. 1. Sferical heterosystem model. 

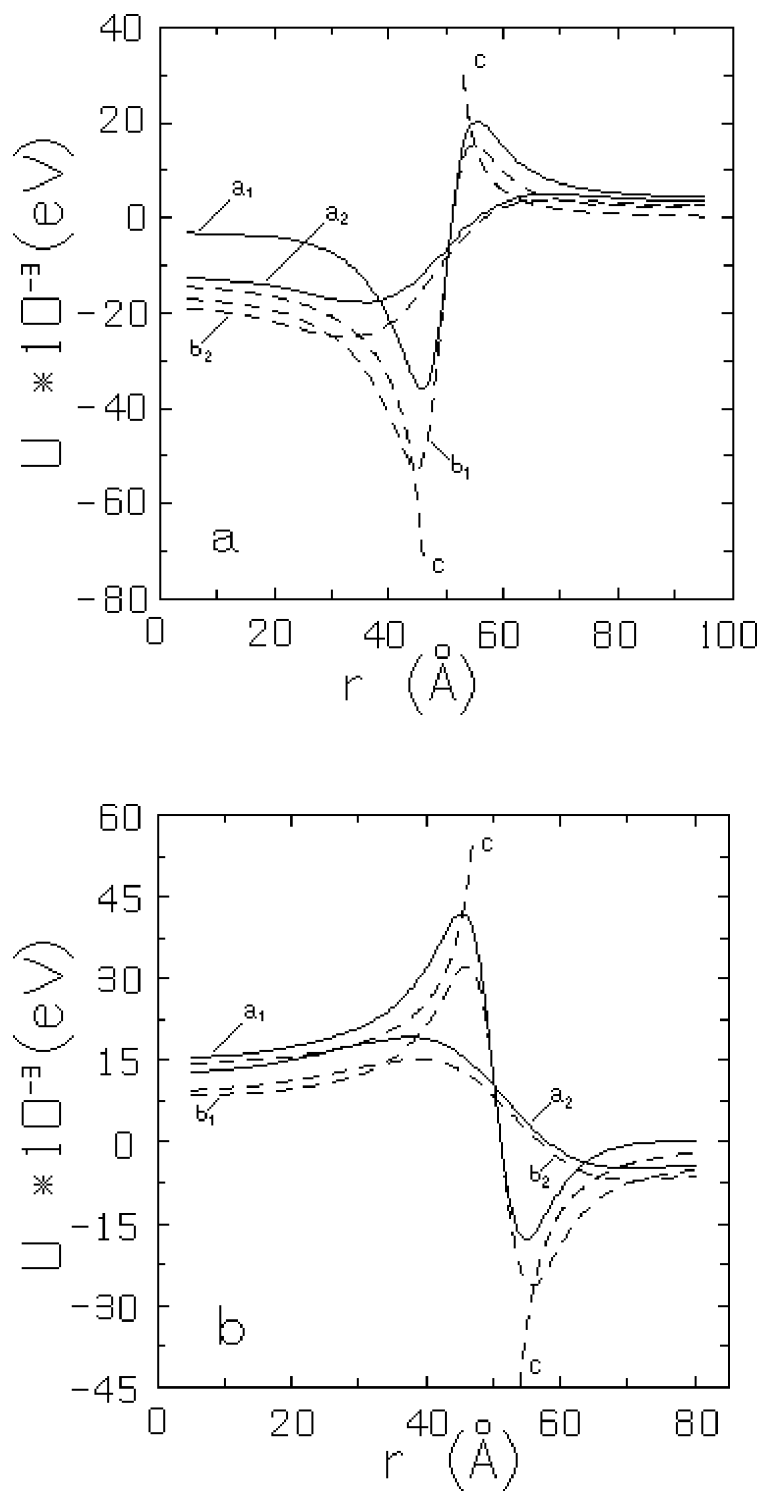

Fig. 2. Potential energy dependence of distance to the centre of microcrystal: the curve a is for total energy of the system, the curve $\mathbf{b}$ shows the contribution of $U_{1}$ term, the curve c represents potential energy at the absence of intermediate layer. Indices " 1 " and " 2 " stand for $L=5 \AA, L=15 \AA$ correspondingly.

\section{STATING THE PROBLEM. THE GENERAL APPROACH}

In a flat interfacial zone, which devides two homogeneous media with dielectric constans $\varepsilon_{1}$ and $\varepsilon_{2}$ and whose thickness is $L$ (Fig. 1), we assume the dielectric constant to be

$$
\varepsilon(r)=\frac{1}{2}\left(\varepsilon_{1}+\varepsilon_{2}\right)\left[1-\gamma \cdot f\left(\frac{r-a}{L}\right)\right],
$$

$$
\begin{aligned}
& f( \pm \infty)= \pm 1, \quad f^{\prime}( \pm \infty)=0, \\
& \gamma=\frac{\varepsilon_{1}-\varepsilon_{2}}{\varepsilon_{1}+\varepsilon_{2}}
\end{aligned}
$$

Let $\phi\left(\mathbf{r}, \mathbf{r}_{0}\right)$ be the induced potential at $\mathbf{r}$ when a point change $q$ is located at position $\mathbf{r}_{0}$. Then $\phi$ proves to satisfy a Poisson's equation

$$
\begin{aligned}
\nabla^{2} \phi\left(\mathbf{r}, \mathbf{r}_{0}\right) & +\frac{d}{d r}(\ln \varepsilon) \cdot \frac{\partial}{\partial r}\left(\phi\left(\mathbf{r}, \mathbf{r}_{0}\right)\right) \\
& =-\frac{4 \pi q}{\varepsilon} \delta\left(\mathbf{r}-\mathbf{r}_{0}\right)
\end{aligned}
$$

with $\delta\left(\mathbf{r}-\mathbf{r}_{0}\right)$ denoting Dirac's delta function. Then in the field induced by a charge its potential energy may be obtained by

$$
U(\mathbf{r})=\frac{q \phi(\mathbf{r})}{2}
$$

where

$$
\phi(\mathbf{r})=\lim _{\mathbf{r} \rightarrow \mathbf{r}_{0}}\left\{\phi\left(\mathbf{r}, \mathbf{r}_{0}\right)-\frac{q}{\varepsilon(r)} \frac{1}{\left|\mathbf{r}-\mathbf{r}_{0}\right|}\right\} .
$$

The function $f(x)$ from Eq. (1) may really attain different forms. Here it is determined by

$$
f(x)=\operatorname{th}(x)
$$

Poisson's Eq. (4) by the substitution

$$
\phi\left(\mathbf{r}, \mathbf{r}_{0}\right)=-4 \pi q\left[\varepsilon(r) \varepsilon\left(r_{0}\right)\right]^{-\frac{1}{2}} G\left(\mathbf{r}, \mathbf{r}_{0}\right)
$$

is reduced to

$$
\nabla^{2} G-V(r) G=\delta\left(\mathbf{r}-\mathbf{r}_{0}\right)
$$

where

$$
V(r)=\frac{1}{2} \frac{1}{r^{2} \sqrt{\varepsilon}} \frac{d}{d r}\left[\frac{r^{2}}{\sqrt{\varepsilon}} \frac{d \varepsilon}{d r}\right]
$$

As seen in Eqs. (1) and (10) value of the image "potential" $V(r)$ depends on $\gamma$. Let us assume that $\varepsilon_{1}$ and $\varepsilon_{2}$ do not differ much. Then $\gamma \ll 1$ and interaction between the interface and charge proves to be weak.

The solution of Eq. (9) has been obtained by approximation method taking account of a small value of the image "potential" $V(r)$. Thus the Green's function can be determined as a rapidly converging series:

$$
G=G^{(0)}+G^{(1)}+G^{(2)}+\ldots \equiv G^{(0)}+\Delta G
$$




\section{I. BOICHUK, R. YU. KUBAY}

In the zeroth aproximation Eq. (9) can be identified

$$
\nabla^{2} G^{(0)}\left(\mathbf{r}, \mathbf{r}_{0}\right)=\delta\left(\mathbf{r}-\mathbf{r}_{0}\right)
$$

$G^{(0)}$ is the potential of a point charge with an accuracy to a constant

$$
G^{(0)}\left(\mathbf{r}, \mathbf{r}_{0}\right)=-\left\{4 \pi\left|\mathbf{r}-\mathbf{r}_{0}\right|\right\}^{-1}
$$

On taking account of higher order corrections consistently we get an arbitrary correction $G^{(n)}$ to satisfy

$$
\nabla^{2} G^{(n)}-V(r) G^{(n-1)}=0
$$

where

$$
\begin{aligned}
G^{(1)}\left(\mathbf{r}, \mathbf{r}_{0}\right) & =\int d \mathbf{r}_{1} G^{(0)}\left(\mathbf{r}, \mathbf{r}_{1}\right) V\left(r_{1}\right) G^{(0)}\left(\mathbf{r}_{1}, \mathbf{r}_{0}\right) \\
G^{(2)}\left(\mathbf{r}, \mathbf{r}_{0}\right) & =\int d \mathbf{r}_{1} d \mathbf{r}_{2} G^{(0)}\left(\mathbf{r}, \mathbf{r}_{1}\right) V\left(r_{1}\right) G^{(0)}\left(\mathbf{r}_{1}, \mathbf{r}_{2}\right) V\left(r_{2}\right) G^{(0)}\left(\mathbf{r}_{2}, \mathbf{r}_{0}\right) \\
G^{(n)}\left(\mathbf{r}, \mathbf{r}_{0}\right) & =\int d \mathbf{r}_{1} \ldots d \mathbf{r}_{n} G^{(0)}\left(\mathbf{r}, \mathbf{r}_{1}\right) V\left(r_{1}\right) \ldots V\left(r_{n}\right) G^{(0)}\left(\mathbf{r}_{n}, \mathbf{r}_{0}\right)
\end{aligned}
$$

With the use of Eqs. (6), (8), (11) and (13) we expand $\phi(r)$ in Eq. (5) by corrections of higher order than of the zeroth

$$
\phi(r)=-\frac{4 \pi q}{\varepsilon(r)} \Delta G(\mathbf{r}, \mathbf{r}),
$$

where

$$
\Delta G(\mathbf{r}, \mathbf{r})=G^{(1)}(\mathbf{r}, \mathbf{r})+G^{(2)}(\mathbf{r}, \mathbf{r})+\ldots
$$

Let us now introduce the Fourier transformations

$$
\begin{aligned}
G^{(0)}\left(\mathbf{r}, \mathbf{r}_{0}\right) & \equiv G^{(0)}\left(\mathbf{r}-\mathbf{r}_{0}\right)=-(2 \pi)^{-3} \int d \mathbf{q} \frac{1}{q^{2}} \exp \left\{i \mathbf{q}\left(\mathbf{r}-\mathbf{r}_{0}\right)\right\} \\
V(r) & =(2 \pi)^{-3} \int d \mathbf{q} V(\mathbf{q}) \exp \{i \mathbf{q r}\}
\end{aligned}
$$

after the substitution into (15) we get

$$
G^{(1)}(\mathbf{r}, \mathbf{r})=(2 \pi)^{-6} \int d \mathbf{q}_{1} d \mathbf{q}_{2} d \mathbf{r}_{1} \frac{V\left(r_{1}\right)}{q_{1}^{2} q_{2}^{2}} \exp \left\{i\left(\mathbf{q}_{1}-\mathbf{q}_{2}\right)\left(\mathbf{r}-\mathbf{r}_{1}\right)\right\}
$$

after integration with respect to $\mathbf{q}_{1}$ and $\mathbf{q}_{2}$ expression (19) is reduced to

$$
G^{(1)}(\mathbf{r}, \mathbf{r})=(4 \pi)^{-2} \int d \mathbf{r}_{1} \frac{V\left(r_{1}\right)}{\left(\mathbf{r}_{1}-\mathbf{r}\right)^{2}} .
$$

Similarly we have

$$
G^{(2)}(\mathbf{r}, \mathbf{r})=-(4 \pi)^{-3} \int d \mathbf{r}_{1} d \mathbf{r}_{2} \frac{V\left(r_{1}\right) V\left(r_{2}\right)}{\left|\mathbf{r}-\mathbf{r}_{1}\right|\left|\mathbf{r}_{1}-\mathbf{r}_{2}\right|\left|\mathbf{r}_{2}-\mathbf{r}\right|},
$$




$$
G^{(n)}(\mathbf{r}, \mathbf{r})=(-4 \pi)^{-(n+1)} \int d \mathbf{r}_{1} d \mathbf{r}_{2} \ldots d \mathbf{r}_{n} \frac{V\left(r_{1}\right) V\left(r_{2}\right) \ldots V\left(r_{n}\right)}{\left|\mathbf{r}-\mathbf{r}_{1}\right|\left|\mathbf{r}_{1}-\mathbf{r}_{2}\right| \cdot \ldots \cdot\left|\mathbf{r}_{n-1}-\mathbf{r}_{n}\right|\left|\mathbf{r}_{n}-\mathbf{r}\right|}
$$

The function $V(r)$ depends only on the absolute value of $r$, therefore we choose the axis to go in the direction of $\mathbf{r}$. After the transition to spherical coordinates and integration over the angular variables we have

$$
G^{(1)}(\mathbf{r}, \mathbf{r})=\frac{1}{8 \pi r} \int_{0}^{\infty} d r_{1} r_{1} V\left(r_{1}\right) \ln \left|\frac{r_{1}+r}{r_{1}-r}\right|
$$

The correction $G^{(2)}(\mathbf{r}, \mathbf{r})$ is obtained in a similar way

$$
\begin{aligned}
G^{(2)}(\mathbf{r}, \mathbf{r}) & =-\frac{1}{8 \pi r} \int_{0}^{r} d r_{1} r_{1} V\left(r_{1}\right) \int_{0}^{r_{1}} d r_{2} r_{2} V\left(r_{2}\right) F\left(\frac{r_{2}}{r}\right)-\frac{1}{8 \pi r} \int_{0}^{r} d r_{1} r_{1} V\left(r_{1}\right) F\left(\frac{r_{1}}{r}\right) \int_{r_{1}}^{r} d r_{2} r_{2} V\left(r_{2}\right) \\
& -\frac{1}{8 \pi r} \int_{0}^{r} d r_{1} r_{1} V\left(r_{1}\right) \int_{r}^{\infty} d r_{2} r_{2} V\left(r_{2}\right) F\left(\frac{r_{1}}{r_{2}}\right)-\frac{1}{8 \pi r} \int_{r}^{\infty} d r_{1} r_{1} V\left(r_{1}\right) \int_{0}^{r} d r_{2} r_{2} V\left(r_{2}\right) F\left(\frac{r_{2}}{r_{1}}\right) \\
& -\frac{1}{8 \pi r} \int_{r}^{\infty} d r_{1} r_{1} V\left(r_{1}\right) F\left(\frac{r}{r_{1}}\right) \int_{r}^{r_{1}} d r_{2} r_{2} V\left(r_{2}\right)-\frac{1}{8 \pi r} \int_{r}^{\infty} d r_{1} r_{1} V\left(r_{1}\right) \int_{r_{1}}^{\infty} d r_{2} r_{2} V\left(r_{2}\right) F\left(\frac{r}{r_{2}}\right),
\end{aligned}
$$

where

$$
F(x)=\int_{0}^{x} d y \frac{1}{y} \ln \frac{1+y}{1-y}, \quad|x| \leq 1
$$

The results obtained above still remain formal. To specify them it is necessary to take account of the explicit form of the function $V(r)$ from Eqs. (10) and (1). Now $V(r)$ becomes as follows

$$
V(r)=-\frac{1}{4 L^{2} r}\left[\frac{4 L \gamma f^{\prime}\left(\frac{r-a}{L}\right)}{1-\gamma f\left(\frac{r-a}{L}\right)}+\frac{2 \gamma r f^{\prime \prime}\left(\frac{r-a}{L}\right)}{1-\gamma f\left(\frac{r-a}{L}\right)}+\left\{\frac{\gamma f^{\prime}\left(\frac{r-a}{L}\right)}{1-\gamma f\left(\frac{r-a}{L}\right)}\right\}^{2} r\right]
$$

The weak interaction is under consideration here $(|\gamma| \ll 1)$. Therefore we expand Eq. (10) with Eq. (1) in power series of $\gamma$. The function $V(r)$ proves to be quite ordinary (we take account of the terms proportional to $\gamma$ only).

$$
V(r) \approx-\frac{\gamma}{L r} f^{\prime}\left(\frac{r-a}{L}\right)-\frac{\gamma}{2 L^{2}} f^{\prime \prime}\left(\frac{r-a}{L}\right)=-\frac{\gamma}{2 r} \frac{d^{2}}{d r^{2}}\left(r f\left(\frac{r-a}{L}\right)\right)
$$

After substituting (27) into (23) and (24) and the corresponding integration carried out $G^{(1)}(\mathbf{r}, \mathbf{r})$ and $G^{(2)}(\mathbf{r}, \mathbf{r})$ are expressed as

$$
\begin{aligned}
G^{(1)}(\mathbf{r}, \mathbf{r}) & =-\frac{\gamma}{8 \pi} \int_{0}^{\infty} d\left(r_{1} f\left(\frac{r_{1}-a}{L}\right)\right) \frac{1}{r_{1}^{2}-r^{2}}, \\
G^{(2)}(\mathbf{r}, \mathbf{r}) & =-\frac{\gamma^{2}}{32 \pi r} \int_{0}^{\infty} d\left(r_{1} f\left(\frac{r_{1}-a}{L}\right)\right) \ln \left|\frac{r_{1}+r}{r_{1}-r}\right| \frac{1}{r_{1}} \frac{d}{d r_{1}}\left(r_{1} f\left(\frac{r_{1}-a}{L}\right)\right) \\
& -\frac{\gamma^{2}}{8 \pi r} \int_{0}^{r} d\left(r_{1} f\left(\frac{r_{1}-a}{L}\right)\right) \int_{r}^{\infty} d\left(r_{2} f\left(\frac{r_{2}-a}{L}\right)\right) \frac{1}{r_{1}^{2}-r_{2}^{2}},
\end{aligned}
$$




\section{I. BOICHUK, R. YU. KUBAY}

In a partial case with a charge placed far from the interfacial zone $\left(\left|r_{1}-a\right| \ll|r-a|\right)$ we get

$$
G^{(1)}(\mathbf{r}, \mathbf{r})=\frac{\gamma}{4 \pi} \frac{a}{r^{2}-a^{2}}, \quad(a \gg L) .
$$

If we substitute Eq. (30) into Eq. (18), and then the result of it into Eq. (5) using Eq. (3) we get the known [10-12] image potential for the spherical interfacial zone

$$
U_{1}(r)=\frac{q^{2}}{2 \varepsilon_{i}}\left(\frac{\varepsilon_{1}-\varepsilon_{2}}{\varepsilon_{1}+\varepsilon_{2}}\right) \frac{a}{a^{2}-r^{2}}, \quad(r \neq a)
$$

where

$$
\varepsilon_{i}= \begin{cases}\varepsilon_{1}, & r<a \\ \varepsilon_{2}, & r>a\end{cases}
$$

The general form of the potential to take account of two corrections of the Green's function will be

$$
U(r)=-\frac{2 \pi q^{2}}{\varepsilon(r)} \Delta G,
$$

where

$$
\Delta G=G^{(1)}+G^{(2)} .
$$

To determine $\Delta G$ we need to discuss a specific form of the function $f=f(x)$.

\section{DISCUSSION}

As is mentioned above, in the ordinary dielectric intermediate layer the function $f=f(x)$ may be determined as

$$
f\left(\frac{r-a}{L}\right)=\operatorname{th}\left(\frac{r-a}{L}\right)
$$

Thus the potential energy of the interaction between the interfacial zone and a charge is as follows

$$
U(r)=U_{1}(r)+U_{2}(r),
$$

where

$$
\begin{aligned}
U_{1}(r) & =\frac{q^{2} \gamma}{4 \varepsilon(r)} \int_{0}^{\infty} d r_{1} \frac{1}{r_{1}^{2}-r^{2}}\left[\frac{r_{1}}{L} \operatorname{sech}^{2}\left(\frac{r_{1}-a}{L}\right)+\operatorname{th}\left(\frac{r_{1}-a}{L}\right)\right], \\
U_{2}(r) & =\frac{q^{2} \gamma^{2}}{16 \varepsilon(r) r} \int_{0}^{\infty} d r_{1} \ln \left|\frac{r_{1}+r}{r_{1}-r}\right| \frac{1}{r}\left[\frac{r_{1}}{L} \operatorname{sech}^{2}\left(\frac{r_{1}-a}{L}\right)+\operatorname{th}\left(\frac{r_{1}-a}{L}\right)\right]^{2} \\
& +\frac{q^{2} \gamma^{2}}{4 \varepsilon(r) r} \int_{0}^{r} d r_{1}\left[\frac{r_{1}}{L} \operatorname{sech}^{2}\left(\frac{r_{1}-a}{L}\right)+\operatorname{th}\left(\frac{r_{1}-a}{L}\right)\right] \int_{r}^{\infty} d r_{2} \frac{1}{r_{1}^{2}-r_{2}^{2}}\left[\frac{r_{2}}{L} \operatorname{sech}^{2}\left(\frac{r_{2}-a}{L}\right)+\operatorname{th}\left(\frac{r_{2}-a}{L}\right)\right] .
\end{aligned}
$$


In Fig. 2(a,b) the dependences $U=U(r)$ (curve a), $U_{1}=U_{1}(r)$ (curve $\mathbf{b}$ ) are given with the parameters of the microcrystal such as $a=50 \AA$, if $L=5 \AA, L=15 \AA$ in the cases

$$
\begin{aligned}
& \text { a) } \quad \varepsilon_{1}=5, \quad \varepsilon_{2}=10 ; \\
& \text { b) } \quad \varepsilon_{1}=10, \quad \varepsilon_{2}=5 .
\end{aligned}
$$

To take account of the existence of polarization charges at the interfacial zone of the media in the case of stepwise change of the dielectric constant results in the potential energy of the charge to be of the Coulomb character [1012] and the function $U=U(r)$ to undergo discontinuity at $r=a$.

If $\varepsilon=\varepsilon(r)$ is described by Eq. (1) or (34) then as can be seen in Fig. 2, the function $U=U(r)$ becomes continuous and changing non-monotonically in the region of the intermediate layer. Besides that the main contribution to the function $U=U(r)$ is given by the term $U_{1}=U_{1}(r)$ to arise due to the first correction of the Green's function. The important point is that a decrease of the thickness of the intermediate layer $(L)$ is followed by a sharper dependence $U=U(r)$ and in the limit $L \rightarrow 0$ we can obtain the result known from [10-12].

In the vicinity of the surface of the crystal placed in a dielectric matrix there is a possibility of bound states of a charge particle to arise. The preceding work [10] dwells on the conditions of existence of the states mentioned. Taking account of the intermediate layer modifies the conditions of both interfacial states of electrons, holes and excitons. These are the problems our further work will focus on

\section{ACKNOWLEDGMENT}

The authors would like to thank I. Shevchuk for helpful discussions.
[1] M. F. Deihen, M. N. Hlinchuk, Fiz. Tverd. Tela 5 (11), 3250 (1963).

[2] I. V. Lerner, Yu. E. Lozovik, Fiz. Tverd. Tela 20 (8), 2241 (1978)

[3] N. M. Niepomniashchii, V. N. Nishanov, Fiz. Tverd. Tela 26 (10), 3184 (1984).

[4] Yu. E. Lozovik, V. N. Nishanov, Fiz. Tverd. Tela 18 (11), 3267 (1976).

[5] V. I. Boichuk, I. V. Bilynsky, Ukr. Fiz. Zh. 35 (10), 1495 (1990).

[6] V. I. Boichuk, I. V. Bilynsky, Phys. Status Solidi 174 (1), 463 (1992).

[7] V. A. Holovatsky, M. V. Tkach, Ukr. Fiz. Zh. 33 (8), 1241 (1988).
[8] V. I. Boichuk, S. M. Kovalchuk, Ukr. Fiz. Zh. 40 (6), 587 (1995).

[9] V. I. Boichuk, O. M. Voitseckhivskaya, V. A. Holovatsky, M. V. Tkach, Fiz. Tverd. Tela 37 (3), 861 (1995).

[10] N. A. Yefriemov, S. I. Pokutnii, Fiz. Tverd. Tela 27 (1), 48 (1985).

[11] N. A. Yefriemov, S. I. Pokutnii, Fiz. Tverd. Tela 32 (10), 2921 (1990).

[12] N. A. Yefriemov, S. I. Pokutnii, Fiz. Tverd. Tela 32 (6), 1637 (1990).

[13] M. V. Tkach, V. I. Boichuk, V. A. Holovatsky, O. M. Voitseckhivskaya, preprint ICMP NAS of Ukraine 95 (6E), 24 (1995).

[14] T. Nakamura, J. Phys. Soc. Jpn. 52 (3), 973 (1983).

\title{
ПОТЕНЦІЯЛЬНА ЕНЕРІІЯ ЗАРЯДУ БІЛЯ ПОВЕРХНІ СФЕРИЧНОГО НАПІВПРОВІДНИКОВОГО МІКРОКРИСТАЛУ ПРИ НАЯВНОСТІ ПРОМІЖНОГО ШАРУ ЗІ ЗМІННОЮ ДІЕЛЕКТРИЧНОЮ ПРОНИКНІСТЮ
}

\author{
В. І. Бойчук, Р. Ю. Кубай \\ Дрогобицький держабний педагогічний університет ім. Івана Франка, кафедра теоретичной фізики \\ вул. Івана Франка, 24, Дрогобич, 82100, Украйна \\ Tel.: (03244)2-20-74, fax: (03244)3-83-76,e-mail:administrator@pedro.uar.net
}

\begin{abstract}
Робота присвядтена дослі,тженню впливу межі поділу напівпровідникової гетеросистеми (сферичний мікрокристал-матриця) на заряджену частинку. Розглянуто випадок, коли біля межі поділу існує проміжний шар, у якому діелектрична проникність є функцією координати. Методом класичних функцій Гріна визначено потенціяльну енергію заряду як функцію відстані до центра мікрокристала для різних діелектричних проникностей гетеросистеми.
\end{abstract}

